\title{
Pacific
}

Journal of

Mathematics

\section{A CLASSIFICATION OF CERTAIN 3-DIMENSIONAL CONFORMALLY FLAT EUCLIDEAN HYPERSURFACES}

OSCAR J. GARAY 


\title{
A CLASSIFICATION OF CERTAIN 3-DIMENSIONAL CONFORMALLY FLAT EUCLIDEAN HYPERSURFACES
}

\author{
OSCAR J. GARAY
}

\begin{abstract}
This paper deals with conformally flat hypersurfaces of the 4dimensional Euclidean space $E^{4}$. We classify those conformally flat hypersurfaces of $E^{4}$ whose mean curvature vector, $H$, is an eigenvector of their Laplacian i.e. $\Delta H=\lambda H ; \lambda \in R$.

The classification is done by proving that the classical CartanSchouten result remains valid for this kind of hypersurfaces.
\end{abstract}

1. Introduction. A Riemannian manifold $\left(M^{n}, g\right)$ is conformally flat, if every point has a neighborhood which is conformal to an open set in the Euclidean space. A submanifold of the Euclidean space $E^{n+1}$ is said to be conformally flat if so it is with respect to the induced Riemannian structure. Thus, in the highest codimension, we can talk about conformally flat hypersurfaces $M^{n}$ of the Euclidean space $E^{n+1}$. We classify conformally flat Euclidean hypersurfaces in $E^{4}$ whose mean curvature vector is an eigenvector of their Laplacian. More concretely we prove that if $x: M \rightarrow E^{4}$ is a complete conformally flat hypersurface immersed in the 4-dimensional Euclidean space, which satisfies $\Delta H=\lambda H, H$ being the mean curvature vector of the immersion, then it is either minimal or the Riemannian product $E^{p} \times S^{(3-p)}, 0 \leq p \leq 3$.

The dimension of the hypersurface seems to play an important role in the study of conformally flat Euclidean hypersurfaces. For $n=2$, the existence of isothermal coordinates means that any Riemannian surface is conformally flat. We shall discuss the case $n=3$ at the end of this section. For $n \geq 4$, the following result of Cartan-Schouten, [2], [27], is of fundamental importance: If $M^{n}$ is a hypersurface immersed in $E^{n+1}, n \geq 4$, then $M^{n}$ is conformally flat in the induced metric, if and only if, at least $n-1$ of the principal curvatures coincide at each point. (See also Nishikawa and Maeda [24].) Using this theorem Kulkarni [20] and Nishikawa and Maeda [24] gave a local classification of conformally flat hypersurfaces in $E^{n+1}, n \geq 4$.

Unfortunately canal hypersurfaces $[4, p .166]$ are conformally flat hypersurfaces which do not fall under this classification. Also, Cecil 
and Ryan, [3], showed that some cyclides of Dupin are counterexamples to both, the mentioned local classification, and to the attempt of Nishikawa [23] of classifying the complete analytic conformally flat hypersurfaces of dimension greater than three in the Euclidean space, $E^{n+1}$.

In [1], D. Blair showed that the generalized catenoid and the hyperplanes are the only conformally flat minimal hypersurface of $E^{n+1}$, $n \geq 4$. Pinl and Ziller also obtained this result in [25] but their proof uses the local classification result of Kulkarni.

In [4, p. 159], Chen defined a locus of $r$-spheres in $E^{m}$ as a submanifold $M$ of $E^{m}$ which is obtained from the smooth glueing of some $n$-dimensional submanifolds of $M$ (possibly with boundary) such that each of the submanifolds is foliated by $r$-spheres $(0<r<n)$ of $E^{m}$. In Theorem 4.4 of [4, p. 165], Chen and Yano gave both local and global descriptions of conformally flat hypersurfaces of $E^{n+1}, n \geq 4$, in terms of loci of $(n-1)$-spheres. In fact, they showed that every conformally flat hypersurface of $E^{n+1}, n \geq 4$, is a locus of $(n-1)$ spheres. Although not every locus of $(n-1)$-spheres is conformally flat in general, Chen and Yano proved in Theorem 4.2 of [4, p. 162] that a locus of $(n-1)$-spheres in $E^{n-1}, n \geq 4$, is conformally flat if and only if the unit normal vector field of the hypersurface, restricted to each of the $(n-1)$-spheres, is parallel with respect to the normal bundle of the $(n-1)$-sphere in $E^{n+1}$. In Theorem 3.1 of [4, p. 157] they gave an intrinsic characterization of conformally flat hypersurfaces.

From the viewpoint of conformal geometry, several important results should be mentioned. Kuiper [19] proved that a compact simply connected conformally flat space, of dimension greater than two, is conformally equivalent to $S^{n}$. On the other hand the topological types of compact conformally flat hypersurfaces in $E^{n+1}, n \geq 4$, are known [16]. The authors describe also in [16] the extrinsic geometry of such hypersurfaces. Finally, Pinkall determines their intrinsic conformal geometry, proving [26, Theorem 2, p. 218]: Every compact conformally flat hypersurface in $E^{n+1}, n \geq 4$, is conformally equivalent to a classical Schottky manifold.

Completeness is not a conformal invariant; therefore, from the conformal geometry point of view, compactness is the simplest global condition that can be imposed on a conformally flat manifold. However, under the Riemannian geometry point of view, completeness has still a role to play. Cecil and Ryan [3] classified the conformally flat hypersurfaces of $E^{n+1}, n \geq 4$, with the additional assumption that the 
immersion is taut (and therefore complete). In [17] we gave a classification of complete conformally flat hypersurfaces of $E^{n+1}, n \geq 4$, using an extra condition on the mean curvature vector, namely, that the mean curvature vector is an eigenvector of the Laplacian of $M^{n}$ with respect to the induced metric. This extrinsic condition forces the manifold into some rather simple shapes.

Leaving aside the result of Kuiper, all the above-mentioned propositions in dimension $n \geq 4$ use the Cartan-Schouten theorem. In other words, they use the fact that for $n \geq 4$ the conformally flat hypersurfaces of $E^{n+1}$ have one principal curvature whose multiplicity is at least $n-1$. Cartan-Schouten's result is no longer true in dimension 3. Lancaster [21, p. 6] gave some examples of conformally flat hypersurfaces in $E^{4}$ having three different principal curvatures.

Probably due to this fact, there are not so many results concerning the classification of conformally flat hypersurfaces in $E^{4}$. Lancaster [21] describes the conformally flat hypersurfaces in $E^{4}$ having diagonal Weingarten map with respect to a conformal coordinate system.

In this paper we use an idea of Chen [11] to prove that the main result in [17] is also true for $n=3$. That is, if $\Delta$ is the Laplacian of $M^{3}$ with respect to the induced metric, and $H$ its mean curvature vector, then we have

THEOREM. Let $M^{3}$ be a complete conformally flat hypersurface of $E^{4}$. Then it satisfies the condition $\Delta H=\lambda H, \lambda \in R$, if and only if $M^{3}$ is one of the following submanifolds

(a) a minimal hypersurface,

(b) a round 3-sphere, $S^{3}(r)$,

(c) a cylinder over a circle $S^{1}(r) \times E^{2}$,

(d) a cylinder over a 2-sphere $S^{2}(r) \times E^{1}$.

The main point in the proof of the above theorem is to show that, if the mean curvature vector is an eigenvector of the Laplacian of $M^{3}$ and if it is not minimal, then the Cartan-Schouten result is still valid. As a consequence we can use the computations of [17] to obtain the result. For conformally flat minimal hypersurfaces in $E^{4}$, we are not able to prove that there is one principal curvature of multiplicity at least two. If this were the case, one might use the method of [1] to prove that $M^{3}$ is either the generalized catenoid or $E^{3}$ (see final remark page 236 of [1]).

This work was done while the author was visiting the Department of Mathematics of Michigan State University, during the first semester of 
1991. I would like to thank my colleagues there, Professors Chen, Blair and Ludden, for their hospitality. I owe special thanks to Professor Chen who brought to my attention his paper [11] and for his comments and suggestions during the preparation of this paper.

2. Main results. Suppose that $M^{3}$ is a connected Riemannian manifold and let $\bar{x}: M^{3} \rightarrow E^{4}$ be an isometric immersion of $M^{3}$ as an orientable hypersurface of $E^{4}$. Let us denote by $\sigma, A, H, \nabla$ and $D$ the second fundamental form, the Weingarten map, the mean curvature vector, the Riemannian connection of $M^{n}$ and the normal connection of the immersion. Suppose that $\xi$ is a global unit normal vector on $M^{n}$ and that $\alpha$ in the mean curvature function with respect to $\xi$, that is $H=\alpha \xi$. The following lemma is a special case of Chen's formula [6], Lemma 4.1, p. 271 (for a proof see [6] and [17]).

LEMma 1 (Chen's formula). Let $\bar{x}: M^{3} \rightarrow E^{4}$ be an orientable hypersurface of $E^{4}$. Then

$$
\Delta H=\left\{\Delta \alpha+\alpha|\sigma|^{2}\right\} \xi+2 A(\nabla \alpha)+\frac{3}{2} \nabla \alpha^{2},
$$

$\Delta$ being the Laplacian of $M^{n}$ in the induced metric and $\nabla \alpha^{2}$ the gradient of $\alpha^{2}$.

Take a local orthonormal frame $\left\{e_{1}, e_{2}, e_{3}, e_{4}\right\}$ such that $\left\{e_{i}\right\}$, $i=1,2,3$, are tangent to $M^{3}$ and $e_{4}=\xi$. Denote by $\left\{w^{i}\right\}_{i=1}^{4}$ the dual frame and by $\left\{w_{i}^{j}\right\}, i, j=1, \ldots, 4$, the connection forms associated to the frame $\left\{e_{1}, e_{2}, e_{3}, e_{4}\right\}$. Let us write the structure equations of $M^{3}$ corresponding to this system

$$
d w_{i}^{n+1}=\sum_{j=1}^{n} w_{i}^{j} \wedge w_{j}^{n+1}, \quad i, j, k=1, \ldots, n .
$$

If we choose a tangent frame $\left\{e_{i}\right\}_{i=1}^{3}$ which diagonalizes the Weingarten map $A e_{i}=\mu_{i} e_{i}, i=1,2,3$, and use the Codazzi equation $\left(\nabla_{X} A\right) Y=\left(\nabla_{Y} A\right) X$ then from (2.2), (2.3) and (2.4) we obtain

$$
\begin{gathered}
e_{i}\left(\mu_{j}\right)=\left(\mu_{i}-\mu_{j}\right) w_{i}^{j}\left(e_{j}\right), \\
\left(\mu_{j}-\mu_{k}\right) w_{j}^{k}\left(e_{i}\right)=\left(\mu_{i}-\mu_{k}\right) w_{i}^{k}\left(e_{j}\right) .
\end{gathered}
$$


Let $R$ and $r$ be the Ricci and scalar curvature of $M^{3}$ respectively. The following tensor is known as the Schouten tensor

$$
L=-R+\frac{r}{4} g
$$

$g$ being the metric tensor on $M$. Assume now that $M^{3}$ is conformally flat. A result of Weyl $[4$, p. 26] states that

$$
\left(\nabla_{Y} L\right)(Z, W)=\left(\nabla_{Z} L\right)(Y, W)
$$

where $W, Y, Z$ are tangent vector fields to $M^{3}$.

We want to prove the following theorems.

Proposition 1. Let $\bar{x}: M^{3} \rightarrow E^{4}$ be an orientable conformally flat hypersurface of $E^{4}$. If $M^{3}$ satisfies $\Delta H=\lambda H, \lambda \in R$, then $M^{3}$ is either minimal or it has a principal curvature of multiplicity at least two at each point.

In other words, this means that under the condition $\Delta H=\lambda H$, the classical Cartan-Schouten theorem remains valid for conformally flat hypersurfaces in $E^{4}$ other than the minimal ones.

The above result leads automatically to

Proposition 2. Assume that $\bar{x}: M^{3} \rightarrow E^{4}$ is a complete orientable conformally flat hypersurface of $E^{4}$. Then it satisfies $\Delta H=\lambda H, \lambda \in$ $R$, if and only if, $M^{3}$ is one of the following submanifolds

(1) a minimal hypersurface in $E^{4}$,

(2) a hypersphere $S^{3}(r)$,

(3) a cylinder over a circle $E^{2} \times S^{1}(r)$,

(4) a cylinder over a 2-sphere $E^{1} \times S^{2}(r)$.

This result extends Theorem 3.2 of [17] to the case of hypersurfaces in $E^{4}$.

We first prove Proposition 2 assuming that Proposition 1 holds. Then we shall prove Proposition 1.

Proof of Proposition 2. Assume that $M^{3}$ is not minimal. Since $M$ has at most two different principal curvatures at each point, one can proceed in the same way as Theorem 3.1 of [17] to conclude that $M^{3}$ is isoparametric.

If $M^{3}$ is isoparametric we can use a well-known result of Segre [28] to conclude that $M^{3}$ is one of the last three manifolds of Proposition 2 . 
Thus our main concern is proving Proposition 1 . We do it in the following way.

Proof of Proposition 1. We denote by $V$ the open set of $M$ where $A$ has three different principal curvatures. We shall prove that $V$ is either empty or minimal.

Suppose that $V$ is not empty. By using the Gauss equation we have

$$
R(Y, Z)=3 \alpha\langle A Y, Z\rangle-\left\langle A^{2} Y, Z\right\rangle \text {; }
$$

therefore from (2.9) and (2.7)

$$
L(Y, Z)=\frac{r}{4}\langle Y, Z\rangle-3 \alpha\langle A Y, Z\rangle+\left\langle A^{2} Y, Z\right\rangle .
$$

Now (2.8) and (2.10) lead in turn to

$$
\begin{aligned}
Y(r) Z-Z(r) Y= & 12\{Y(\alpha) A Z-Z(\alpha) A Y\} \\
& +4\left\{\left(\nabla_{Z} A^{2}\right) Y-\left(\nabla_{Y} A^{2}\right) Z\right\} .
\end{aligned}
$$

From (2.9) and Codazzi's equation

$$
r=9 \alpha^{2}-|A|^{2} \text {. }
$$

Combining (2.11) and the connection equations we get

$$
\begin{gathered}
\left(\mu_{j}^{2}-\mu_{i}^{2}\right) w_{i}^{j}\left(e_{j}\right)=3 e_{i}(\alpha) \mu_{j}-\frac{1}{4}\left(e_{i} r\right)-e_{i}\left(\mu_{j}^{2}\right), \\
\left(\mu_{j}^{2}-\mu_{k}^{2}\right) w_{j}^{k}\left(e^{i}\right)=\left(\mu_{i}^{2}-\mu_{k}^{2}\right) w_{i}^{k}\left(e_{j}\right)
\end{gathered}
$$

for distinct $i, j, k \in\{1,2,3\}$.

If $V \neq \varnothing$ then we use (2.6) and (2.14) to obtain

$$
w_{i}^{k}\left(e_{j}\right)=0
$$

for distinct $i, k, j \in\{1,2,3\}$. Using Lemma 1 and the hypothesis $\Delta H=\lambda H$ we have

$$
\Delta(\alpha)=\left(\lambda-|\sigma|^{2}\right) \alpha
$$

on $M$ and, in particular, on $V$.

Suppose that $\alpha$ is non-zero constant on some open set $V_{1} \subset V$. We shall see that this is not possible. In fact, we see from (2.16) that $|\sigma|^{2}$ is also constant and, therefore, (2.12) means that $r$ is constant. Then we can use (2.13) to obtain

$$
e_{i}\left(\mu_{j}^{2}\right)=\left(\mu_{i}^{2}-\mu_{j}^{2}\right) w_{i}^{j}\left(e_{j}\right), \quad i \neq j .
$$

Combining (2.5) and (2.17) we get $e_{i}\left(\mu_{j}\right)=0, i \neq j, i, j \in\{1,2,3\}$. But $\alpha$ is constant and then $e_{i}\left(\mu_{i}\right)=0, i \in\{1,2,3\}$. Thus $\mu_{i}$, 
$i=1,2,3$, are constant. From (2.17), $w_{i}^{j}\left(e_{j}\right)=0, i \neq j$, and then using (2.15) $w_{i}^{j}=0$ on $V_{1}$, this means that $V_{1}$ is flat. But then from Gauss equation we have $\mu_{i} \mu_{j}=0, i \neq j$, on $V_{1}$ so that at least two principal curvatures are zero on $V_{1}$ which contradicts $V_{1} \subset V$.

We deduce from the above reasoning that $\alpha$ can't be a non-zero constant on any open set $V_{1} \subset V$.

The following lemma follows easily from Chen's formula (Lemma $1)$.

Lemma 2. Suppose that $\bar{x}: M^{3} \rightarrow E^{4}$ is a hypersurface of $E^{4}$ satisfying $\Delta H=\lambda H$. Then $A\left(\nabla \alpha^{2}\right)=-\frac{3}{2} \alpha\left(\nabla \alpha^{2}\right)$ on the open set $U=\left\{p \in M ; \nabla \alpha^{2}(p) \neq 0\right\}$.

If $V \cap U=\varnothing$ then $\nabla \alpha^{2}=0$ on $V$ means that $\alpha^{2}$ is constant on $V$ and then $\alpha=0$ on $V$. If $V \cap U \neq \varnothing$, we can choose an open set $V_{2} \subset V \cap U$. Now, using Lemma 2 we can take a local orthonormal basis in $V_{2},\left\{e_{1}, e_{2}, e_{3}, e_{4}\right\}$ such that $\left\{e_{1}, e_{2}, e_{3}\right\}$ are tangent vectors to $M^{3}$ diagonalizing $A, A e_{i}=\mu_{i} e_{i}, i=1,2,3, e_{1}$ is parallel to $\nabla \alpha^{2}$ and $e_{4}=\xi$, the normal unit vector. Therefore there exists a function $\delta$ on $V_{2}$ (or on some suitable open set contained in $V_{2}$ ) such that

$$
\mu_{1}=-\frac{3}{2} \alpha ; \quad \mu_{2}=\frac{9}{4} \alpha+\delta ; \quad \mu_{3}=\frac{9}{4} \alpha-\delta .
$$

Thus from (2.5) and because $e_{1} \| \nabla \alpha^{2}$ we get

$$
\begin{aligned}
e_{2}(\alpha) & =e_{3}(\alpha)=0, \\
e_{1}\left(\mu_{2}\right) & =-\left(\delta+\frac{15}{4} \alpha\right) w_{1}^{2}\left(e_{2}\right), \\
e_{1}\left(\mu_{3}\right) & =\left(\delta-\frac{15}{4} \alpha\right) w_{1}^{3}\left(e_{3}\right) .
\end{aligned}
$$

Since $\mu_{1}=-\frac{3}{2} \alpha$, then $e_{2}\left(\mu_{1}\right)=e_{3}\left(\mu_{1}\right)=0$ and then from (2.5) we have

$$
w_{2}^{1}\left(e_{1}\right)=0=w_{3}^{1}\left(e_{1}\right) .
$$

Therefore using (2.15) and (2.20) we obtain

$$
w_{1}^{2}=\phi w^{2} ; \quad w_{1}^{3}=\eta w^{3}
$$

where $\phi=w_{1}^{2}\left(e_{2}\right)$ and $\eta=w_{1}^{3}\left(e_{3}\right)$. Now from (2.18) and (2.19)

$$
\phi=-\frac{9 e_{1}(\alpha)+4 e_{1}(\delta)}{4 \delta+15 \alpha} ; \quad \eta=\frac{9 e_{1}(\alpha)-4 e_{1}(\delta)}{4 \delta-15 \alpha} .
$$


Differentiating $w_{1}^{4}=\mu_{1} w^{1}$ and considering (2.18) and (2.21), we have $d w_{1}^{4}=0$ and therefore, locally, there exists a function $u$, such that $w^{1}=d u$. Then, by using (2.19), $d \alpha \wedge d u=0$ and thus $\alpha$ is a function of $u$.

Combining now (2.5) and (2.13) we get

$$
3 e_{i}(\alpha) \mu_{j}-\frac{1}{4} e_{i}(r)=\left(\mu_{j}-\mu_{i}\right) e_{i}\left(\mu_{j}\right) .
$$

From this equation and (2.12) we obtain

$$
e_{i}\left(\mu_{j}\right)\left(\mu_{j}-\mu_{i}\right)+\sum_{\substack{h \neq k \neq s \\ h, k, s \in\{1,2,3\}}}\left\{\frac{1}{2}\left(\mu_{k}+\mu_{s}\right)-\mu_{j}\right\} e_{i}\left(\mu_{h}\right)=0 .
$$

If we put $i=2 ; j=1$ and $i=3 ; j=1$ in (2.24) we obtain $e_{2}\left(\delta^{2}\right)=e_{3}\left(\delta^{2}\right)=0$ respectively. Since $\mu_{i} \neq \mu_{j}, i \neq j$, in $V_{2}$, we have $\delta \neq 0$, and therefore $e_{2}(\delta)=e_{3}(\delta)=0$. Thus $\delta$ depends on $u, \delta(u)$. We write $\alpha^{\prime}, \delta^{\prime}$ for $e_{1}(\alpha)$ and $e_{1}(\delta)$ respectively. Putting $i=1, j=2$ in (2.24) we get $\delta \alpha^{\prime}=5 \alpha \delta^{\prime}$; hence

$$
\alpha=c \delta^{5}, \quad c \in R \text {. }
$$

We can now combine (2.25), (2.22), (2.19) and (2.18) to obtain

$$
\begin{gathered}
\mu_{1}=-\frac{3}{2} c \delta^{5} ; \quad \mu_{2}=\frac{9}{4} c \delta^{5}+\delta ; \quad \mu_{3}=\frac{9}{4} c \delta^{5}-\delta \\
w_{1}^{2}=-\frac{\left(45 c \delta^{4}+4\right) \delta^{\prime}}{\left(4+15 c \delta^{4}\right) \delta} w^{2} ; \quad w_{1}^{3}=\frac{\left(45 c \delta^{4}-4\right) \delta^{\prime}}{\left(4-15 c \delta^{4}\right) \delta} w^{3} .
\end{gathered}
$$

We know that $e_{i}(\alpha)=e_{i}(\delta)=0, i=1,2$; then from (2.19) $e_{i}\left(\mu_{j}\right)=0, i, j=2,3$. Using (2.13) we get $e_{1}\left(\mu_{1}\right)=e_{2}\left(\mu_{1}\right)=0$ and thus $e_{i}(r)=0$. Using this fact in (2.13) and (2.14) we have

$$
\left(\mu_{3}^{2}-\mu_{2}^{2}\right) w_{2}^{3}\left(e_{3}\right)=0,
$$$$
\left(\mu_{2}^{2}-\mu_{3}^{2}\right) w_{3}^{2}\left(e_{2}\right)=0 \text {. }
$$

If $\mu_{3}^{2}=\mu_{2}^{2}$, then either $\mu_{3}=\mu_{2}$ or $\mu_{3}=-\mu_{2}$ the first case is clearly impossible because we are in $V$. If $\mu_{3}$ were equal to $-\mu_{2}$, then using (2.26) we have $\delta=0$, and therefore from (2.25) $\alpha=0$ on $V_{2}$. If $\mu_{3}^{2} \neq \mu_{2}^{2}$, then from (2.28) and (2.15) we have

$$
w_{2}^{3}=0 \text {. }
$$

Taking differentiation of the second equation of (2.27) and using (2.26) and (2.29) we get

$$
\begin{aligned}
\left(45 c \delta^{4}+4\right)\left(4 \delta+15 c \delta^{5}\right) \delta^{\prime \prime} & \\
= & \left(2700 c^{2} \delta^{8}-60 c \delta^{4}+32\right)\left(\delta^{\prime}\right)^{2} \\
& -\frac{3}{2} c \delta^{8}\left(\frac{9}{4} c \delta^{4}+1\right)\left(4+15 c \delta^{4}\right)^{2}
\end{aligned}
$$


Analogously differentiating the first equation of (2.27) and using (2.26) and (2.29) one has

$$
\begin{aligned}
\left(45 c \delta^{4}-4\right)\left(4 \delta-15 c \delta^{5}\right) \delta^{\prime \prime} \\
=-\left(2700 c^{2} \delta^{8}+60 c \delta^{4}+32\right)\left(\delta^{\prime}\right)^{2} \\
\quad+\frac{3}{2} c \delta^{8}\left(\frac{9}{4} c \delta^{4}-1\right)\left(4-15 c \delta^{4}\right)^{2}
\end{aligned}
$$

Combining (2.30) and (2.31), we find

$$
\begin{aligned}
& \left(1377200 c^{2} \delta^{8}+17280\right)\left(\delta^{\prime}\right)^{2} \\
& \quad=\frac{3}{8} \delta^{4}\left(1701000 c^{4} \delta^{16}+149760 c^{2} \delta^{8}-1024\right) .
\end{aligned}
$$

Now taking differentiation of (2.29) and using (2.26) and (2.27) we have

$$
\begin{aligned}
& 16\left(2025 c^{2} \delta^{8}-16\right)\left(\delta^{\prime}\right)^{2} \\
& \quad=\delta^{4}\left(-18225 c^{4} \delta^{16}+4896 c^{2} \delta^{8}-256\right) .
\end{aligned}
$$

Finally, from (2.32) and (2.33) we can conclude that $\delta$ is constant on $V_{2}$. Therefore by (2.25) $\alpha$ is constant on $V_{2}$ which implies that $\alpha=0$ on $V_{2}$. Since $V_{2}$ is arbitrary, $\alpha=0$ on $V \cap U$, but clearly $\alpha=0$ on $V \cap\left(M^{3}-U\right)$; consequently it is zero on $V$. Hence either $V=\varnothing$ or it is minimal.

Suppose that $V=\varnothing$; then $M^{3}$ has at most two different principal curvatures. On the other hand, if $V$ is minimal then it is contained in the set $W=\left\{p \in M^{3} / \alpha(p)=0\right\}$. Consider the open submanifold $W_{1}=M^{3}-W$. Then there are at most two different principal curvatures on $W_{1}$ and using the method of Theorem 3.1 of [17] we see that it is isoparametric and therefore it has constant mean curvature $\alpha$. By continuity, $\alpha=0$ on $M^{3}$, that is, $M^{3}$ is minimal.

\section{Final remarks.}

REMARK 3.1. B. Y. Chen and K. Yano introduced in 1972 the notion of quasiumbilical submanifolds [4, p. 147]. An isometric immersion $\bar{x}: M^{n} \rightarrow E^{m}$ in the Euclidean space is quasiumbilical with respect to a unit normal direction $\xi$, if the Weingarten map associated to $\xi, A_{\xi}$, admits an eigenvalue of multiplicity at least $n-1$. The immersion is said to be (totally) quasiumbilical if for every point $p \in M^{n}$ there exists a local orthonormal basis of $(T M)^{\perp}$, $\left\{\xi_{n+1}, \ldots, \xi_{m}\right\}$, such that $\left(M^{n}, \bar{x}\right)$ is quasiumbilical with respect to $\xi_{j}, j=n+1, \ldots, m$.

In terms of quasiumbilical submanifolds, the Cartan-Schouten result can be rephrased in the following way: An Euclidean hypersurface 
$M^{n}, n \geq 4$, is conformally flat, if and only if it is quasiumbilical. Chen and Yano [4, Prop. 1.11, p. 148] proved that every quasiumbilical Euclidean submanifold $M^{n}, n \geq 3$, is conformally flat. The converse of this result is not true in general [21]. For $n>3$ and under suitable restrictions on the codimension of $M^{n}$ the converse also holds [14], [22]. For a detailed study see [29].

Our Proposition 1 can be interpreted in the light of quasiumbilicity condition: A conformally flat non-minimal hypersurface $M^{3}$ or $E^{4}$ satisfying $\Delta H=\lambda H$ is quasiumbilical.

REMARK 3.2. There is also a close link between submanifolds satisfying $\Delta H=\lambda H$ and finite type submanifolds [6], [7]. Indeed, if $\lambda=0$, then $\Delta H=0$ and therefore $\Delta^{2} \bar{x}=0$. These submanifolds are called biharmonic and have been studied by several authors [8], [12], [15]. Chen and Ishikawa [12] and Dimitric [15], proved that a biharmonic submanifiold is either minimal or of infinite type. In fact, in many cases, the only possibility is the minimal one. For instance Chen in 1985 proved that a biharmonic surface in $E^{3}$ is minimal. Also Dimitrić [15] showed that a biharmonic submanifold $M$ of the Euclidean space is minimal in the following cases: (a) $M$ is a curve, (b) $M$ has constant mean curvature or (c) $M$ is a hypersurface with at most two distinct principal curvatures. In [9] B. Y. Chen made the following conjecture: the only biharmonic submanifolds in the Euclidean space are the minimal ones. The conjecture is true for spherical submanifolds [9].

If $\delta H=\lambda H$ with $\lambda \neq 0$, then Chen [13] has proved that they are either of 1-type or of null 2-type. Summarizing this result, we have that given an Euclidean isometric immersion $\bar{x}: M^{n} \rightarrow E^{m}$ satisfying $\Delta H=\lambda H$, then if $\lambda=0$, it is either minimal or of infinite type, and if $\lambda \neq 0$, then it is either of 1-type or of null 2-type. Consequently, if the above Chen conjecture is true, the problems of classifying Euclidean submanifolds satisfying $\Delta H=\lambda H$ and that of classifying null 2-type Euclidean submanifolds are basically the same.

REMARK 3.3. If $\bar{x}: M^{n} \rightarrow E^{m}$ is an isometric immersion and $\Delta$ represents the Laplacian with respect to the induced metric, then the following Beltrami equation is well known: $\Delta \bar{x}=-n H, H$ being the mean curvature vector of the immersion. In particular the above formula says that the Laplacian of the position vector of the immersion is everywhere perpendicular to $M^{n}$.

The family of submanifolds satisfying $\Delta H=\lambda H$ that we have studied before verifies also that the Laplacian of the mean curvature vec- 
tor is everywhere perpendicular to $M^{n}$. In other words, the second Laplacian of the position vector is a normal vector to $M^{n}$. It makes sense therefore to pose the following problem: to study Euclidean submanifolds whose second Laplacian of the position vector (that is the Laplacian of the mean curvature vector) is everywhere perpendicular to $M^{n}$.

We proved in [18] that if $\Delta H=c, c$ being a constant normal vector, then $c=0$, that is $\left(M^{n}, \bar{x}\right)$ is biharmonic. Under the assumption that $\Delta H$ is everywhere normal to $M^{n}$, the main point is that Lemma 2 is still valid no matter what the dimension of $M^{n}$. This gives us important information about one of the principal curvatures. If in addition there are so many principal curvatures, then this information can be crucial. For instance, following the computations of Theorem 3.1 in [17], one can easily deduce the following result.

Proposition 3. Let $M^{n}$ be a conformally flat orientable hypersurface of $E^{n+1}, n>3$. Assume that the Laplacian of the mean curvature vector, $\Delta H$, is everywhere perpendicular to $M^{n}$. Then the mean curvature function $\alpha$ is harmonic, if and only if, it is constant.

Proof. Take $U=\left\{p \in M^{n} ; \nabla \alpha^{2}(p) \neq 0\right\}$. Suppose $\alpha$ is harmonic, if $\alpha$ is not constant, then under the above conditions $\alpha$ must satisfy locally the equation (3.16) of [17], on some open set contained in $U$

$$
\left(\alpha^{\prime}\right)^{2}=c \alpha^{2(n+5) /(n+2)}-\left(\frac{n(n+2)}{2(n-1)}\right)^{2} \alpha^{4}
$$

where $c$ is a constant. On the other hand since $\alpha$ is harmonic, we have from equation (3.17) of [17] that $\alpha$ also satisfies

$$
-(n+2) \alpha \alpha^{\prime \prime}+3(n-1)\left(\alpha^{\prime}\right)^{2}=0 \text {. }
$$

From these equations, we see that $\alpha$ is locally constant in $U$. This is impossible. Therefore $\alpha$ is constant on $M^{2}$.

The conformally flat condition in the above proposition is only needed to assure the existence of at most two different principal curvatures at each point of $M^{n}$. Therefore one can also prove, following the computations of [10], that

Proposition 4. Let $\bar{x}: M^{2} \rightarrow E^{3}$ be an isometric immersion of a surface in $E^{3}$ satisfying that $\Delta H$ is everywhere perpendicular to 
$M^{2}$. Then the mean curvature function is harmonic, if and only if it is constant.

\section{REFERENCES}

[1] D. Blair, A generalization of the catenoid, Canad. J. Math., 27 (1975), 231-236.

[2] E. Cartan, La déformation des hypersurfaces dans l'espace conforme reel a $n \geq 5$ dimensions, Bull. Soc. Math. France, 45 (1917), 57-121.

[3] T. E. Cecil and P. J. Ryan, Conformal geometry and the cyclides of Dupin, Canad. J. Math., 32 (1980), 767-782.

[4] B. Y. Chen, Geometry of Submanifolds, Marcel Dekker, New York (1973).

[5] _ 2-type submanifolds and their applications, Chinese J. Math., 14 (1986), $1-14$.

[6] _ Total mean curvature and submanifolds of finite type, World Scientific Publ., (1984).

[7] _ Finite type submanifolds and generalizations, University of Rome, Rome, (1985).

[8] __ Some open problems and conjectures on finite type submanifolds, Michigan State University, (1989).

[9] _- Some open problems and conjectures on submanifolds of finite type, Soochow J. Math., 17 (1991), 169-188.

[10] _ Null 2-type surfaces in $E^{3}$ are circular cylinders, Kodai Math. J., 11 (1988), 295-299.

[11] __ Local rigidity theorems of 2-type hypersurfaces in a hypersphere, Nagoya Math. J., 122 (1991).

[12] B. Y. Chen and S. Ishikawa, Biharmonic surfaces in pseudo-Euclidean spaces, Memoirs of the Faculty of Science. Kyushu Univ. Ser. A, 45 (1991), 323-347.

[13] B. Y. Chen, Null 2-type surfaces in Euclidean space, Algebra, Analysis and Geometry, World Scientific, 1988, pp. 1-18.

[14] B. Y. Chen and L. Verstraelen, A characterization of totally quasiumbilical submanifolds and its applications, Boll. Un. Mat. Ital. A(5), 14 (1977), 49-57.

[15] I. Dimitrić, Quadratic representations and submanifolds of finite type, Doctoral Thesis, Michigan State University, (1989).

[16] M. DoCarmo, M. Dajczer and F. Mercuri, Compact conformally flat hypersurfaces, Trans. Amer. Math. Soc., 288 (1985), 189-203.

[17] A. Ferrandez, O. J. Garay and P. Lucas, On a certain class of conformally flat Euclidean hypersurfaces, Proc. Conference on Global Analysis and Global Differential Geometry, Lecture Notes in Math., vol. 1481, Springer-Verlag, Berlin and New York, 1990, pp. 48-54.

[18] O. J. Garay and A. Romero, An isometric embedding of the complex hyperbolic space in a pseudo-Euclidean space and its application to the study of real hypersurfaces, Tsukuba J. Math., 14 (1990), 293-313.

[19] N. H. Kuiper, On conformally flat spaces in the large, Ann. of Math., 50 (1949), 916-924.

[20] R. S. Kulkarni, Conformally flat manifolds, Proc. Nat. Acad. Sci. U.S.A., 69 (1972), 2675-2676.

[21] G. M. Lancaster, Canonical metrics for certain conformally Euclidean spaces of three dimension and codimension one, Duke Math. J., 40 (1973), 1-8. 
[22] J. D. Moore and J. M. Morvan, Sous-variétés conformément plates de codimension quatre, C. R. Acad. Sci. Paris, 287(A) (1978), 655-657.

[23] S. Nishikawa, Conformally flat hypersurfaces in a Euclidean space, Tôhoku Math. J., 26 (1974), 563-572.

[24] S. Nishikawa and Y. Maeda, Conformally flat hypersurfaces in a conformally flat manifold, Tôhoku Math. J., 26 (1974), 159-168.

[25] M. Pinl and W. Ziller, Minimal hypersurfaces in spaces of constant curvature, J. Differential Geom., 11 (1976), 335-343.

[26] U. Pinkall, Compact conformally flat hypersurfaces, Conformal Geometry, A publication of the Max Planck Institut für Mathematik, R. S. Kulkarni and U. Pinkall (Eds), Bonn, (1988).

[27] J. A. Schouten, Uber die Konforme Abbildung n-dimensionaler Mannigfaltigkeiter mit quadratischer Maß bestimmung auf eine Mannigfaltigkeit mit euklidischer Mßß bestimmung, Math. Z., 11 (1921), 58-88.

[28] B. Segre, Famiglie di ipersuperficie isoparametrische negli spazi euclidei ad un qualungue numero di dimensioni, Atti Acad. Naz. Lincei Rend. Cl. Sci. Fis. Mat. Natur., 27 (1938), 203-207.

[29] G. M. Zafindratafa, Sous-varietes soumises a des conditinos de courbure, Doctoral thesis, Katholieke Universiteit Leuven, (1991).

Received February 3, 1992 and in revised form November 20,1992. Research partially supported by a DGICYT grant PB90-0014-C03-02, Spain.

Departamento de Geometria y Topologia

Facultad de Ciencias

UNIVERSIDAD DE GRANADA

18071 GRANADA

SPAIN 



\title{
PACIFIC JOURNAL OF MATHEMATICS
}

Founded by

\author{
E. F. BeCKenBACH (1906-1982) F. Wolf (1904-1989)
}

\section{EDITORS}

Sun-Yung A. Chang

(Managing Editor)

University of California

Los Angeles, CA 90024-1555

chang@math.ucla.edu

\section{F. Michael Christ}

University of California

Los Angeles, CA 90024-1555

christ@math.ucla.edu

Herbert Clemens

University of Utah

Salt Lake City, UT 84112

clemens@math.utah.edu
THOMAS ENRIGHT

University of California, San Diego

La Jolla, CA 92093

tenright@ucsd.edu

Nicholas ERCOLANI

University of Arizona

Tucson, AZ 85721

ercolani@math.arizona.edu

R. FINN

Stanford University

Stanford, CA 94305

finn@gauss.stanford.edu

VAUghan F. R. Jones

University of California

Berkeley, CA 94720

vfr@math.berkeley.edu
STEVEN KERCKHOFF

Stanford University

Stanford, CA 94305

spk@gauss.stanford.edu

Martin ScharLemanN University of California Santa Barbara, CA 93106 mgscharl@math.ucsb.edu

Harold Stark

University of California, San Diego La Jolla, CA 92093

V. S. VARADARAJAN University of California

Los Angeles, CA 90024-1555

vsv@math.ucla.edu

\section{SUPPORTING INSTITUTIONS}

UNIVERSITY OF ARIZONA

UNIVERSITY OF BRITISH COLUMBIA

CALIFORNIA INSTITUTE OF TECHNOLOGY

UNIVERSITY OF CALIFORNIA

UNIVERSITY OF MONTANA

UNIVERSITY OF NEVADA, RENO

NEW MEXICO STATE UNIVERSITY

OREGON STATE UNIVERSITY
UNIVERSITY OF OREGON

UNIVERSITY OF SOUTHERN CALIFORNIA

STANFORD UNIVERSITY

UNIVERSITY OF HAWAII

UNIVERSITY OF UTAH

WASHINGTON STATE UNIVERSITY

UNIVERSITY OF WASHINGTON 


\title{
PACIFIC JOURNAL OF MATHEMATICS
}

\author{
Volume $162 \quad$ No. $1 \quad$ January 1994
}

Semisimplicity of restricted enveloping algebras of Lie superalgebras $\quad 1$

JEFFERY MARC BERGEN

A classification of certain 3-dimensional conformally flat Euclidean hypersurfaces

OSCAR J. GARAY

Braided groups of Hopf algebras obtained by twisting

D. GUREVICH and SHAHN MAJID

Flat connections, geometric invariants and the symplectic nature of the 45 fundamental group of surfaces

K. GURUPRASAD

Spin models for link polynomials, strongly regular graphs and Jaeger's 57 Higman-Sims model

PIERRE DE LA HARPE

On the uniqueness of representational indices of derivations of $C^{*}$-algebras

EDWARD KISSIN

On infinitesimal behavior of the Kobayashi distance

MYUNG YULL PANG

Vertex operator construction of standard modules for $A_{n}^{(1)}$

MIRKO PRIMC

Bergman and Hardy spaces with small exponents

KEHE ZHU 